\section{Verein zur Förderung der klinischen Verhaltenstherapie e. V. (VFkV)}

VFkV, Ausbildungsinstitut München, Lindwurmstr. 117, D-80337 München, Tel. +49 89 834-6900, Fax -8659

\section{Nachruf: Dr. med. Dieter Schwarz}

Am 13.03.2003 verstarb Dr. Dieter Schwarz, Arzt für Neurologie und Psychiatrie. Sein berufliches Leben war geprägt von einem außergewöhnlichen Interesse an therapeutischem Denken und Handeln, welches sich bis in die letzten Wochen nach der Diagnose einer schweren Krebserkrankung zeigte. Bis wenige Tage vor seinem Tod supervidierte er junge Kolleginnen und Kollegen und betreute seine Patienten.

Sein Name verbindet sich mit der ersten verhaltenstherapeutischen Klinik und dem ersten Ausbildungsinstitut für Verhaltenstherapie in Deutschland. Die Psychosomatische Klinik Windach leitete er als Chefarzt von 1976 bis 1990 und gab damit einen wichtigen Anstoß für die Etablierung der Verhaltenstherapie in der Gesundheitsversorgung in Deutschland. Er bildete die erste Generation von Ärzten und Psychologen aus, die nicht primär über Forschung auf die Verhaltenstherapie gestoßen waren, sondern eine Optimierung der Versorgung von kranken Menschen zum Ziel hatten. 1983 erfolgte die Gründung des ersten Ausbildungsinstitutes für Verhaltenstherapie zur Erlangung der Kassenzulassung für Psychologen in Deutschland, der Verein zur Förderung der klinischen Verhaltenstherapie (VFkV) in München. Er wirkte als Dozent, Seminarleiter sowie Supervisor und bereicherte Vorstandssitzungen unermüdlich mit Anregungen.

Dieter Schwarz wurde am 05.03.1935 in Königsberg/Ostpreußen geboren. Die Flucht führte die Familie über viele Stationen nach Bad Godesberg. Dort legte er das Abitur ab und studierte dann Psychologie, Philosophie, Anglistik, Germanistik und Theaterwissenschaften in Bonn, Frankfurt am Main und Freiburg bei Adorno, Horkheimer und Heidegger. 1957 entschied er sich für das Medizinstudium in Bonn, legte 1963 das Staatsexamen ab und promovierte über «unspezifische Anpassungsprozesse». Nach der Medizinalassistentenzeit bewarb er sich 1966 erfolgreich bei Professor Ploog am neu gegründeten Max-Planck-Institut für Psychiatrie in München. Dort erwarb er Erfahrungen und Grundwissen der Verhaltenstherapie und erweiterte diese durch Auslandsaufenthalte.
Zusammen mit anderen entstand die Idee einer verhaltenstherapeutisch orientierten Klinik. Neu war nicht nur die therapeutische Ausrichtung, sondern auch die Gestaltung der inneren Strukturen des Krankenhauses vor dem Hintergrund des theoretischen Konzeptes. In vielen psychosomatischen Kliniken sind diese Änderungen heute eine Selbstverständlichkeit (Rolle der Ko-Therapeuten, feste Bezugstherapeuten für Patienten, keine Visiten am Krankenbett, stattdessen ausführliche Besprechungen und Supervisionen, medizinische Zentralen usw.). Neu war auch in Deutschland ein gleichberechtigtes Team aus Ärzten und Psychologen. Verhaltenstherapeutisch arbeitenden Psychologen öffnete dies den Weg zur Krankenbehandlung.

Für seine Mitarbeiter war Dieter Schwarz früher Chef und Ausbilder, dann Supervisor und Mentor und schließlich ein in verlässlicher Freundschaft zugewandter Kollege. Er faszinierte mit seiner intellektuellen Brillanz und seiner weit gefächerten Bildung. Im Kontakt mit ihm und in seinem Kontakt mit Patienten wurde eine ausgeprägte Menschenliebe spürbar, von Neugier und Erkenntnisdrang geprägt, im Umgang voll unaufdringlicher Zuwendung, ohne Alters- und Standesgrenzen. Er liebte das gesprochene und geschriebene Wort, den Disput auch um des Disputes Willen, das Experiment und die Literatur.

Dieter Schwarz war nicht nur ein bedeutender Arzt. Möglicherweise wichtiger ist sein Einfluss auf die vielen Psychotherapeuten, denen er seinen pragmatischen und kreativen Therapiestil vermittelt hat. Ohne persönliche Eitelkeit verstand er es, eine anregende und geistig offene Atmosphäre zu schaffen, die einen starken Zusammenhalt unter den Mitarbeitern erzeugte, eigene Stärken bewusst werden und Vertrauen in das eigene - nicht nur therapeutische - Handeln gewinnen ließ.

Seine schwere Erkrankung hat ihn körperlich geschwächt, innerlich wirkte er jedoch stark und strahlte Ruhe und Gelassenheit aus.

Viele trauern um den verehrten Lehrer und Freund - wir sind ihm zutiefst dankbar.

Der Vorstand des Ausbildungsinstitutes München im Verein zur Förderung der klinischen Verhaltenstherapie, VFkV, sowie seine ärztlichen und psychologischen Kollegen und Mitarbeiter 


\section{Deutsche Gesellschaft für Verhaltens- medizin und Verhaltensmodifikation (DGVM)}

Schriftführerin der DGVM: Prof. Dr. Ulrike Ehlert, Psychologisches Institut der Universität Zürich, Klinische Psychologie II, Zürichbergstrasse 43, CH-8044 Zürich Tel. +411 634-30 97, Fax -3696

E-Mail schriftfuehrerin@dgvm-online.de,

Homepage www.dgvm-online.de

\section{Bericht der Arbeitsgruppe «DRGs» (Kooperation unterschiedlicher Fachgesellschaften aus dem Bereich der psychosozialen Medizin mit Beteiligung der DGVM, vertreten durch U. Cuntz): Unsichere Prognosen für existentielle Fragen: Die Psychosomatik und die DRGs}

Ohne Zweifel ist der im internationalen Vergleich hohe Entwicklungsstand der stationären Psychosomatik und psychotherapeutischen Medizin in Deutschland begünstigt durch ein Finanzierungssystem, das eine Entgeltregelung für psychotherapeutische Leistungen nicht nur für die stationäre Rehabilitation, sondern auch im Bereich der Akutkrankenhäuser ermöglicht. In den letzten zwei Jahren erlebten alle, die diesen hohen Standard der Versorgung aufrechterhalten wollen oder deren berufliche Zukunft von der Entgeltregelung für die Krankenhäuser abhängt, und alle, die sich mit der Finanzierungsregelung für die stationäre Psychosomatik und psychotherapeutische Medizin beschäftigten, ein Wechselbad der Gefühle.

In den ersten Entwürfen des Fallpauschalengesetzes, das ja die Einführung einer Entgeltregelung entsprechend der australischen DRGs für weite Bereiche der deutschen Krankenhausversorgung vorsieht, war - wie in Australien auch - die Psychiatrie von dieser Regelung ausgenommen. Dagegen sollte die Psychosomatik eingeschlossen werden, obwohl die australischen DRGs auch diesen Bereich (der in Australien auch Teil der psychiatrischen Versorgung ist) nicht adäquat abbilden. Fast in letzter Minute wurde bei der schließlich verabschiedeten Fassung des Fallpauschalengesetzes die Psychosomatik ausgenommen. Dennoch bestand die Absicht, diesen Teil des Gesundheitssystems möglichst rasch in die DRG-Regelung zu integrieren, zumal Teile der psychosomatischen Betten zu internistischen Abteilungen gehören, die in Zukunft nach DRGs abrechnen müssen. Bis heute besteht für diese Betten keine adäquate Regelung.

Ende letzten Jahres wurde deutlich, dass sich das DRG-System keineswegs so reibungslos von Australien nach Deutschland transplantieren lässt, wie ursprünglich erhofft. Für viele Bereiche besteht ein ganz erheblicher Nachbesserungsbedarf, und es ist zu erwarten, dass eine «Scharfstellung» des Systems, die ja für 2006 projektiert ist, nur mit zahlreichen Sonderregelungen möglich ist.
Die bisherige Anpassung des Systems wurde weitestgehend ohne den Sachverstand der betroffenen Ärzte und Therapeuten durch die Selbstverwaltungsorgane der Krankenkassen und der Deutschen Krankenhausgesellschaft durchgeführt. Die Lücken und Unzulänglichkeiten des Systems werden allerdings erst dann wirklich transparent, wenn die Auswirkung der DRGs auf die Patientenversorgung im konkreten Einzelfall unter Berücksichtigung der tatsächlich vorliegenden Diagnosen des Patienten bewertet wird. Die Politik hat jetzt einen entscheidenden Schritt auf die Fachgesellschaften hin gemacht und sie ganz offen zur Mitarbeit aufgefordert. In einem ersten Schritt wurde den Fachgesellschaften die Gelegenheit gegeben, bis zum 31.03.2003 Vorschläge zur Revision des DRG-Systems beim Deutschen DRG-Institut (InEK) einzureichen.

Die (zahlreichen) Fachgesellschaften für den Bereich Psychosomatik und psychotherapeutische Medizin (DGVM, Ä̈GP, DGPM, DÄVT, DKPM) hatten bereits vorher eine verbandsübergreifende Arbeitsgruppe gebildet. Dieser Gruppe ist es unter anderem gelungen, den Prozedurenkatalog, der ein wesentlicher Bestandteil des DRG-Systems sein wird, um psychotherapeutische Ziffern zu erweitern. Die Arbeitsgruppe ermöglicht es den Fachgesellschaften, mit einer Stimme zu sprechen und nicht durch widersprüchliche Stellungnahmen den Einfluss gegenseitig zu mindern. Anlässlich des DGVMKongresses in Göttingen fand ein Treffen dieser Arbeitsgruppe statt, bei der folgende Stellungnahme verabschiedet und an das InEK weitergeleitet wurde:

\section{Vorschlag der verbandsübergreifenden Arbeitsgruppe Psychosomatische und psychotherapeutische Medizin zum DRG-System}

Im australischen System gibt es keine Fallkostenkalkulation für stationäre Psychosomatik und psychotherapeutische Medizin

Stationäre Psychotherapie spielt bei den australischen DRGs nur eine sehr untergeordnete Rolle für die errechneten Fallkosten. Folgerichtig kann das australische System keine geeignete Grundlage für eine Entgeltregelung in der hoch differenzierten stationären psychosomatischen und psychotherapeutischen Versorgung in Deutschland sein.

\section{Die Diagnosengruppierung im australischen System ist nicht geeignet, um Fallkosten für die stationäre Psychotherapie zu differenzieren}

Die in den australischen DRGs zusammengefassten Diagnosen der MDC 19 (mental health) sind weder nach inhaltlichen noch nach ökonomischen Kriterien sinnvoll zusammengefasst. Offensichtlich (dies kann aus den in Australien bei diesen Diagnosegruppen eingesetzten Prozeduren geschlossen werden) werden diese DRGs zur somatischen diagnostischen Abklärung und in geringerem Umfang auch zur Krisenintervention 
abgerechnet. Eine mit kurativer Intention eingesetzte stationäre Psychotherapie wird in Australien nur im Rahmen der psychiatrischen Versorgung angeboten, die dort komplett aus dem DRG-System herausgenommen ist. Daher ist eine Neugruppierung der MDC19 notwendig (s.u.).

\section{Beispiele}

U65Z Angststörungen: umfasst neben den «phobischen Störungen» (F40.-) und den «anderen Angststörungen» (F41.-) auch die «Konversionsstörung» (F44.8) und «Schlafstörungen»(G47.-). Inhaltlich ist diese Diagnosegruppierung nur sinnvoll, wenn damit die somatische Differentialdiagnostik oder kurz dauernde Behandlung von «Erregungszuständen» unterschiedlicher Genese gemeint ist. Damit ist jedoch auf keinen Fall die Behandlung der Störungen selbst gemeint. Um das in der deutschen Psychosomatik und psychotherapeutischen Medizin wesentlich besser ausgereifte Therapiespektrum darzustellen, ergibt sich die Notwendigkeit einer ganz anderen Gruppierung, in der Angststörungen und Schlafstörungen auf keinen Fall gemeinsam in einer Gruppe stehen können.

U66Z Essstörungen: umfasst neben der «Anorexia nervosa» (F50.0) auch die «Bulimia nervosa» (F50.2) und die «nicht näher bezeichneten Essstörungen» (F50.9). Darüber hinaus wurden dieser DRG auch die «Zwangsstörungen» (F42.8) zugeordnet, was unter dem Gesichtspunkt des einheitlichen Ressourcenverbrauchs inhaltlich nicht stimmig ist. Der notwendige therapeutische Aufwand, der sich bei diesen Diagnosen in psychosomatischen und psychotherapeutischen Krankenhäusern ergibt, ist so unterschiedlich, dass diese Art der Gruppierung keinesfalls die Fallkosten abbilden kann. Die Anorexia nervosa braucht je nach Schweregrad (der am einfachsten über den Body Mass Index (BMI) bei Aufnahme zu definieren ist) die intensivste Therapie, während bei manchen anderen Essstörungen je nach Schweregrad eine ambulante Behandlung ausreicht. Zwangsstörungen benötigen (auch hier nach Schweregrad sehr divergierend) eine spezielle Behandlung, die bezüglich des Aufwandes nicht mit der der Essstörungen gleichgesetzt werden kann.

U67Z Persönlichkeitsstörungen und akute Reaktionen: umfasst u.a. «Anpassungsstörungen» (F43.2), die «posttraumatische Belastungsstörung» (F43.1) und die «Borderline Persönlichkeitsstörung»(F60.3). Auch alle anderen Persönlichkeitsstörungen werden dieser DRG zugeordnet. Bei dieser DRG ist aus Sicht der Psychosomatik und psychotherapeutischen Medizin keinerlei inhaltliche Stimmigkeit zu erkennen. Neben leichten Störungen, die nur als Zusatzdiagnosen relevant sind oder auch ambulant behandelt werden können, finden sich mit den emotional instabilen und Borderline Persönlichkeitsstörungen auch Erkrankungen, die in therapeutischen Spezialeinrichtungen einer langwierigen und aufwändigen stationären Fachbehandlung bedürfen.

\section{Die G-DRGs bilden die Fallkosten der stationären}

\section{Psychotherapie weder in den Kostengewichten noch in der}

\section{Diagnosegruppierung adäquat ab}

Da die Einführung der G-DRGs vollständig auf den australischen Fallgruppen beruht und die errechneten Kostengewichte offensichtlich ausschließlich die Kosten für Krisenintervention und Ausschlussdiagnostik abbilden, sind sie in der vorliegenden Form in keiner Weise geeignet, die Leistungsstrukturen der psychosomatisch/psychotherapeutischen Einrichtungen abzubilden.

Da für die meisten der aufgeführten Diagnosen auch keine sinnvolle Behandlung in den bereits im DRG-System befindlichen Krankenhäusern angeboten wird, schlagen wir die völlige Streichung der MDC 19 vor. Sie muss ersetzt werden durch eine neue Diagnosegruppierung, in der die Kosten für eine etwaige somatische Diagnostik und für Krisenintervention bei psychischen Störungen erfasst werden, und einer davon verschiedenen Gruppierung, die die Kosten für die stationäre Psychosomatik und Psychotherapie abdeckt. Die Kosten sind wesentlich von der Art der notwendig werdenden diagnostischen und therapeutischen Prozeduren bestimmt (vgl. OPS 301.2, Ziff. 9-402.0) und diese sind für Ausschlussdiagnostik und Krisenintervention vollständig andere als bei stationären Behandlungen, die nach den Regeln der Psychosomatik und psychotherapeutischen Medizin durchgeführt werden. Eine einheitliche Fallgruppierung, die sowohl die Ausschlussdiagnostik als auch Krisenintervention und die stationäre Psychotherapie abbildet, ist deswegen nicht möglich.

ICD-10 SGB V keine ausreichende Basis für valide Diagnosen im Bereich der Psychosomatik und psychotherapeutischen Medizin/Psychiatrie (Verzicht auf deskriptive Kriterien)

Die Diagnosen des Kapitels «Psychische und Verhaltensstörungen» sind deskriptiv definiert, d.h. sie können nur dann gestellt und extern überprüft werden, wenn eine vorgegebene Anzahl von Kriterien erfüllt werden. Die den G-DRG zugrunde gelegte Version der ICD-10 SGB V verzichtet auf die Nennung dieser Kriterien. Damit fehlt eine verlässliche Basis für die Abrechnung der DRGs. Für diesen Bereich der ICD10 sollten deswegen die wissenschaftlichen Kriterien der ICD10 gelten, damit nicht dem upcoding Tür und Tor geöffnet wird.

\section{Hohe Kostenrelevanz der Prozeduren}

Stationäre Psychosomatik und psychotherapeutische Medizin sind personalaufwändig. Die Personalkosten sind der bei weitem dominierende Teil der Fallkosten. Um zu verhindern, dass unzureichende Therapieprogramme genauso vergütet werden wie die notwendig intensiven, hoch frequenten Programme spezialisierter Kliniken, muss die Abrechnung der entsprechenden DRGs an die im OPS 301.2 notwendige Mindesttherapiedosis (9-402.0 Psychosomatische und psychotherapeutische Komplexbehandlung) geknüpft sein. Nur so kann auch dauerhaft 
ein qualitativ hoch stehendes Therapieangebot für Patienten mit psychischen Störungen gesichert werden. Denkbar sind DRGs, die analog zu den operativen Fächern an die Erbringung einer definierten therapeutischen Leistung geknüpft sind.

\section{Ausreichende Homogenität der Fallkosten}

Unter der Voraussetzung, dass die Fallkosten in leistungsfähigen Krankenhäusern bzw. Abteilungen für Psychosomatik und psychotherapeutische Medizin erhoben werden und dass Fallgruppen gebildet werden, die sich an diesen Fallgruppen und dem für die darin enthaltenen Diagnosen notwendigen Therapieangebot (Therapiedosis pro Zeit) orientieren, ist davon auszugehen, dass die Homogenität der Fallkosten ausreicht, um eine Abrechung nach (neu zu erstellenden) DRGs auch in der Psychosomatik und psychotherapeutischen Medizin zu gewährleisten. - Die diesen Vorschlag unterstützenden AWMF-Fachgesellschaften der verbandsübergreifenden AG sind jederzeit zu einer entsprechenden Kooperation mit dem Ziel einer Fallkostenkalkulation bereit.

\section{Qualitätssicherung (sowohl Struktur-als auch Ergebnis-} qualität) ist die Voraussetzung für ein funktionierendes DRGSystem in der Psychosomatik

Die Erfahrungen anderer Länder mit DRG-gebundener Entgeltregelung zeigen, dass ein hohes Risiko darin besteht, günstige Abrechnungsbilanzen durch die Absenkung der Qualität und der Therapiedauer zu erreichen. Um dies zu verhindern, muss die Einführung der DRGs für die Psychosomatische und psychotherapeutische Medizin flankiert werden durch qualitätssichernde Maßnahmen. Diese sollten sich auf Zuweisungskriterien, Diagnostik, Therapieintensität und -dauer so wie vor allem auch die Ergebnisqualität beziehen. Ohne diese Maßnahmen entsteht mit den DRGs ein ökonomischer Anreiz für schlechtere Qualität. - Die diesen Vorschlag unterstützenden AWMF-Fachgesellschaften der verbandsübergreifenden $A G$ haben schon vor Jahren ein Qualitätssicherungsprogramm entwickelt und publiziert (z.B. das der Hardcopy-Version beigelegte Psy-BaDo-Manual [Heuft, Senf [Hrsg]. Thieme, Stuttgart] zur Qualitätssicherung in der Psychotherapie), die heute in den qualifizierten Kliniken routinemäßig eingesetzt werden.

\section{Psychosomatische bzw. psychische Komorbidität in der Somatik ist kostenrelevant für andere DRG-Bereiche, findet mangels ausreichender Diagnostik jedoch keinen ausreichenden Niederschlag in den PCCLs}

Eine internistische Krankenhausabteilung müsste eigentlich nach Abschluss der stationären Diagnostik einen relevanten Prozentsatz sog. «funktioneller Störungen» (ICD-10 F45.- somatoforme Störungen) als Hauptdiagnose ausweisen - diese liegen in der stationären somatischen Therapie je nach Fach bei bis zu $20-40 \%$ der Patienten vor. Diese wären, wie oben ausgeführt, über eine neu gestaltete MDC 19 abzurechnen. Fehlt dieser Prozentsatz, ist mit einer «Verschleierung» dieser Diagnosen zu rechnen - zu Lasten der Krankenkassen, die auch nach der Entlassung des Patienten aus der stationären Behandlung mit weiterer (unnötiger) somatischer Diagnostik und späterer (ggf. erneuter) Fehlbelegung rechnen müssen. Ein funktionierender psychosomatisch-psychotherapeutischer Konsiliar- und Liaisondienst kann diese Differentialdiagnostik substantiell absichern helfen.

Darüber hinaus ist davon auszugehen, dass Patienten mit somatischer Grunderkrankung, die zusätzlich unter einer psychosomatischen bzw. psychischen Komorbidität leiden, einen höheren Ressourcenverbrauch induzieren.

\section{Beispiele}

- Patient mit einem schweren Unfalltrauma und einer zusätzlichen posttraumatischen Belastungsstörung (PTBS; ICD-10: F43.1)

- Patient mit organisch bedingter Epilepsie in Kombination mit dissoziativen Anfällen (ICD-10: F44.-)

- Tumorpatient mit schweren Problemen der Krankheitsverarbeitung im Sinne einer Anpassungsstörung (ICD-10: F43.2-)

In diesen Fällen fehlt jede Schweregradsteigerung durch Kodierung der psychosomatischen bzw. psychischen Komorbidität, deren Diagnosequalität auf Facharztniveau durch dokumentierte ICD-10-Kriterien (Checklisten liegen vor) sichergestellt werden kann. Viele Krankenhäuser verfügen noch nicht über einen psychosomatisch-psychotherapeutischen Konsiliarund Liaisondienst, und das G-DRGs «bestraft» die Kliniken, die entsprechend qualifiziert arbeiten, dadurch, dass die Kostenrelevanz der psychosomatischen bzw. psychischen Komorbidität unberücksichtigt bleibt. In den bisherigen Kalkulationsmodellen wird die Kostenrelevanz dieser psychosomatischen bzw. psychischen Komorbidität nicht erfasst, da sie als Kriterium in den Kodierungen nicht oder nur sehr marginal abgebildet werden. Wie durch epidemiologische Studien hinlänglich abgesichert, entspricht dies in keiner Weise der - auch ökonomischen - Bedeutung dieser Störungen.

Die diesen Vorschlag unterstützenden AWMF-Fachgesellschaften der verbandsübergreifenden $\mathrm{AG}$ sind sofort bereit, die ökonomische Bedeutung psychosomatischer bzw. psychischer Komorbidität im Rahmen von Modellprojekten empirisch zu belegen.

Kontaktadressen:

Dr. med. Dipl.-Psych. U. Cuntz

Medizinisch-Psychosomatische Klinik Roseneck

Am Roseneck 6, D-83209 Prien am Chiemsee

Tel. +49 8051 68-3524, Fax -3532

E-mail: UCuntz@Schoen-Kliniken.de

\section{Prof. Dr. Gereon Heuft}

Klinik und Poliklinik für Psychosomatik und

Psychotherapie der Universität Münster

Domagkstr. 11, D-48149 Münster

Tel. +49 251 83-52902 (Sekretariat)

E-mail: heuftge@medsnt01.uni-muenster.de 


\section{Arbeitsgemeinschaft für Verhaltensmodifikation e.V. (AVM)}

Bundesgeschäftsstelle: Dr.-Haas-Str. 4, D-96047 Bamberg, Tel. 0951/20820-39, Fax -49, E-mail info@avm-d.de, Internet www.avm-d.de

Neu im Programm der AVM ist die zertifizierte Zusatzausbildung/Fortbildung zum/r «speziellen Schmerz-Psychotherapeuten/in». Die Zertifizierung erfolgt gemäß den Richtlinien für die Zusatzausbildung in psychologischer Schmerztherapie der Deutschen Gesellschaft für psychologische Schmerztherapie und -forschung (DGPSF) und der Deutschen Gesellschaft zum Studium des Schmerzes (DGSS). Angesprochen werden alle interessierten Diplom-Psychologen/innen, Ärzte/innen für Psychiatrie und Psychotherapie bzw. Psychotherapeutische Medizin und Ärzte/innen mit Zusatzbezeichnung Psychotherapie.

Gesamtumfang der Zusatzausbildung: 80 Stunden Curriculum, 25 Stunden Einzelsupervision, Teilnahme an 24 Sitzungen einer interdisziplinären Schmerzkonferenz, 10 Kurzdokumentationen von supervidierten Behandlungen mit Leitsymptom Schmerz, 6-monatige psychotherapeutische Tätigkeit in oder 2-jährige enge Kooperation mit einer schmerzbezogenen Einrichtung, Abschlusskolloquium.

Kosten:

80-Stunden-Curriculum: $€$ 960,- (AVM-Mitglieder: $€ 880,-)$ 25 Stunden Einzelsupervision: ca. $€ 1550$

Abschlusskolloquium/Zertifizierungsgebühr: $€ 150$

Das Zertifikat «spezielle Schmerz-Psychotherapie» erhalten nach Abschluss der gesamten Zusatzausbildung alle approbierten Diplom-Psychologen/innen und Ärzte/innen mit abgeschlossener Psychotherapie-Ausbildung. Alle anderen Teilnehmer bekommen eine Bescheinigung über Art und Umfang der absolvierten Elemente der Zusatzausbildung.

Informationen über anerkannte Supervisoren, schmerztherapeutische Einrichtungen für die praktische Tätigkeit sowie über interdisziplinäre Schmerzkonferenzen werden im Rahmen des Curriculums bekannt gegeben.
Termine des 80-Stunden-Curriculums: 27.09. - 28.09.03 Bad Mergentheim, Deutschland

Einheit 1: Grundlagen: Psychosoziale Grundlagen des chronischen Schmerzes; Methoden: Hypnotherapeutische Verfahren bei chronischem Schmerz; Anwendung: Kopf- und Gesichtsschmerz. (Dozenten: Dipl.-Psych A. Stenzel, Dipl.-Psych. M. Baierl)

\subsection{6. - 29.06.2003 Bamberg, Deutschland}

Einheit 4: Methoden: Kognitiv-behaviorale Schmerzinterventionen/lösungsfokussierte Verfahren; Anwendung: Somatoforme Schmerzstörung. (Dozenten: Dipl.-Psych. T. Rausch, Dipl.-Psych. T. Tonhauser)

\subsection{7. - 06.07.2003 Bad Mergentheim, Deutschland}

Einheit 2: Grundlagen: Biologische Grundlagen chronischer Schmerzen; Methoden: Medizinische Diagnostik und Therapie/tiefenpsychologische Ansätze; Anwendung: Viszerale und Tumorschmerzen. (Dozent: Dr. med. Dipl.-Psych. C. Derra)

\subsection{8. - 31.08.2003 Bayreuth, Deutschland}

Einheit 3: Methoden: Psychologische Schmerzanamnese und -diagnostik/Klassifikation und Dokumentation/Therapieplanung und -evaluation. (Dozent: Dipl.-Psych. F. Schuchmann)

\subsection{0. - 12.10.2003 Bad Aibling, Deutschland}

Einheit 5: Methoden: Biofeedback, integrative Kurzeittherapie, Physiotherapie und Feldenkrais; Anwendung: Rückenschmerz und Rheumaschmerz. (Dozenten: Dipl.-Psych. U. Hron, Dr. med. A. Leidiger, H. Witte)

\subsection{1. - 08.11.2003 Staffelstein, Deutschland}

Einheit 6: Methoden: Gruppenedukationsprogramme/Entspannungsverfahren; Anwendung: Entzugsbehandlung/altersabhängige Schmerzsyndrome. (Dozentin: Dipl.-Psych. C. Schrembs) 